\title{
Revisiting Blacklisting and Justifying the Unit Disk Graph Model for Energy-Efficient Position-Based Routing in Wireless Sensor Networks
}

\author{
Anthony Kleerekoper \\ School of Computer Science \\ University of Manchester \\ Manchester, M13 9PL, UK \\ Email:kleereka@cs.manchester.ac.uk
}

\author{
Nick Filer \\ School of Computer Science \\ University of Manchester \\ Manchester, M13 9PL, UK \\ Email: nick@cs.manchester.ac.uk
}

\begin{abstract}
The unit disk graph model is widely used in conjunction with position-based routing. Past research has suggested that this leads to inefficient routing strategies and improved metrics have been proposed that combine packet reception rate and progress. In this paper we revisit the earlier analysis and show that a blacklisting scheme based on packet reception rate is more energy efficient. We also show that the unit disk graph model is a close approximation to this energy efficient routing scheme.

Index Terms-blacklisting, geographic routing, position-based routing, radio, unit disk graph model, wireless communication, wireless sensor networks,
\end{abstract}

\section{INTRODUCTION}

A common paradigm for routing in wireless sensor networks is to use position-based routing (also known as geographic routing) with greedy forwarding [1], [2]. Nodes are aware of the locations of their neighbours and forward packets based on progress towards the packet's final destination. The unit disk graph (UDG) model is widely used in sensor network research. It states that any node within a fixed radius $R$ around a transmitting node has a perfect link to the transmitter and all other nodes have no link. That is, the packet reception rate (PRR) of a link shorter than $R$ is $100 \%$, otherwise it is $0 \%$. When the UDG model is used with position-based routing the conclusion is that the only factor a node should consider when choosing a relay is the progress, i.e. how much closer the packet gets to its final destination.

Later researchers found that in reality links show wide variation in PRR in the so-called "transitional region" as shown in Fig. 1 [3]. Based on this observation, it was shown that progress-only metrics have poor performance in the real world because long links are likely to be inside the transitional region and may have low PRR resulting in frequent retransmissions. The conclusions drawn were that PRR should be combined with progress in the cost function and that the UDG model should be avoided. In this paper that analysis is revisited and we show that PRR should be considered but separately from progress. Rather, PRR should be used initially to blacklist links and then progress only should be used to select between the non-blacklisted options. We call this Absolute Receptionbased Blacklisting (ARB). We also show that the UDG model can be a close approximation of the behaviour of ARB.

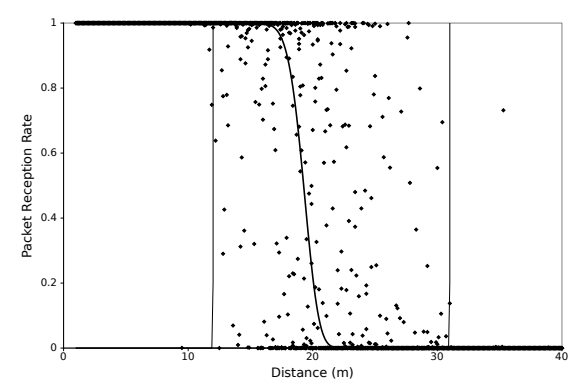

Fig. 1. In the real world three distinct regions exist around a transmitting node each displaying different behaviours of the packet reception rate (PRR). However, the expected PRR (the line) acts very similarly to the UDG model.

\section{RELATED WORK}

Zuniga and Krishnamachari argue that the UDG model relating PRR to distance is too simplistic and propose a more accurate model [3]. Their model, given in equation (1), calculates the signal to noise ratio $(\gamma(d))$ based on the lognormal shadowing model. The constants in the equation are specific to non-coherent FSK and $f$ is the number of bits in the packet.

$$
\operatorname{PRR}(d)=\left(1-\frac{1}{2} \exp ^{-\frac{\gamma(d)}{2} \frac{1}{0.64}}\right)^{8 f}
$$

Seada et al. used this model for analysing the trade-off between distance and PRR of links [1]. Long hops have the advantage of reducing the total number of hops that the packet must traverse but each link may have a low PRR resulting in retransmissions. On the other hand, shorter hops are more likely to have high PRR and fewer retransmissions but more hops are needed. They argue that when selecting the relay node both the progress made and the PRR of the link must be considered together. 
They considered the two cases of networks with and without ARQ and concluded that in both cases the most energyefficient approach to position-based forwarding is to select the link that maximises the function PRR $\times$ distance. Lee et al. generalised the combination of link cost and progress by proposing the normalised advance framework [4]. The idea is that for energy-efficiency, the cost of a link must be normalised against the progress made towards the destination by selecting that link. The same proposal was made independently by Stojmenovic et al. called cost per progress [5].

\section{REVISITING BLACKLISTING}

The original analyses by Seada et al. considered ARQ to be a network-wide choice that it is either used everywhere in the network or nowhere. In both cases they showed that combining PRR and progress into a single metric was more energy efficient than alternative approaches including blacklisting.

However, we argue that ARQ should be considered a function of the links as well as the network. The purpose of ARQ is to ensure that a desired link quality, as measured by the number of successfully received packets, is achieved. Let us suppose that this link quality is $q$ then it is obvious that if the PRR of the link is below the threshold, ARQ is required for all packets. Regardless of the value of $q$ (i.e. even if it is less than 1 and so not all failed transmissions need to be repeated), acknowledgements are needed for all packets so that some failed transmissions can be repeated to raise the effective quality of the link above the threshold.

However, if the chosen link has a PRR greater than $q$ then ARQ is not needed. Although without ARQ it is not possible to know whether the transmission was successful or not, this information is not needed. By definition, if the PRR of the link is above the threshold then even if a packet is not received no retransmission will be needed because overall the proportion of packets being successfully received is above the desired threshold.

Based on this observation we argue that the cost of a link varies according to its PRR and that therefore the cost of the high quality links were overestimated by Seada et al. who did not consider PRR in the cost of a link.

Let $e_{t x}$ and $e_{r x}$ be the energy cost of transmitting and receiving a data packet respectively. Then let $\alpha$ be the relative size of an acknowledgement to a data packet, which generalises from previous works which have assumed that acknowledgements are the same size as data packets [2], [5]. The total cost of using a given link is therefore given by equation (2):

$$
C= \begin{cases}\frac{q}{P R R(d)}\left(e_{t x}+(1+\alpha) e_{r x}\right)+\alpha e_{t x} & P R R(d)<q \\ e_{t x}+e_{r x} & \text { otherwise }\end{cases}
$$

Equation (2) suggests that below-threshold links impose significantly higher costs than other links and that they should therefore be avoided even if they result in more progress being made. This is the absolute reception-based blacklisting scheme (ARB) in which all links with below-threshold PRR are excluded and then, of the remaining links, the one that results in the greatest progress is selected.

As a first indication that this hypothesis is correct a simple chain topology was analysed numerically. A transmitting node is simulated at one end of a $40 \mathrm{~m}$ long chain. The nodes in the chain are set at half meter intervals. For each chain the optimal link is the one that minimises the cost per progress where cost is defined according to equation (2). The variable values used were the same as those used by Zuniga and Krishnamachari [3] and we set $e_{t x}=16.5$ and $e_{r x}=9.6$ as in the CC1000 transceiver.

The values of $\alpha$ and $q$ were varied and for each configuration 10,000 sample chains were generated. Fig. 2 shows that the proportion of optimal links whose PRR was above the threshold rises quickly to close to $100 \%$ indicating that the larger the acknowledgements the more important it is to use only above-threshold links.

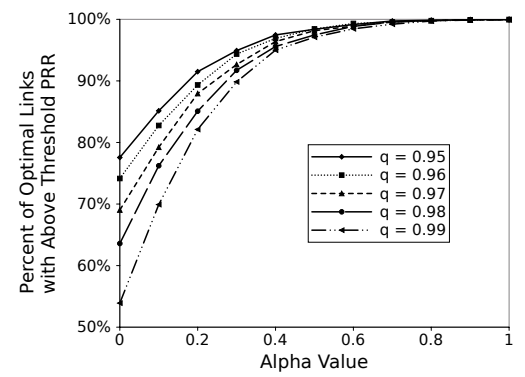

Fig. 2. The more costly acknowledgements are, the more likely it is that the optimal links will have above threshold PRR values.

For a direct comparison with the alternative $\mathrm{PRR} \times$ distance metric a complete network of $100 \mathrm{~m} \times 100 \mathrm{~m}$ was simulated in which nodes were randomly distributed. In each simulation, 100 packets were sent from a source to a destination, with a new source and destination randomly selected for each packet. $q$ is set to one to ensure delivery of every packet.

Fig. 3 shows the average energy consumption along each packet's route for both $\mathrm{ARB}$ and $\mathrm{PRR} \times$ distance for varying network density. For convenience, density is defined in terms of the expected distance between neighbouring nodes. That is, density of $2 \mathrm{~m}$ suggests that the distance between each node and its nearest neighbour is $2 \mathrm{~m}$ which, in a $100 \mathrm{~m} \times 100 \mathrm{~m}$ network, results in 2500 nodes. The results show that ARB consumes less energy than PRR $\times$ distance. The improvement is between $26 \%$ and $51 \%$.

\section{JUSTIFying THE UNIT DISK GRAPH MODEL}

The UDG model is underpinned by two assumptions. The first is that all links are either perfect or non-existent ( $\mathrm{PRR}=100 \%$ or $0 \%$ ). The second is that the cross-over point between the two is a fixed radius. The ARB strategy is effectively the same as the first of these two assumptions in that it treats all links as either being perfect (no need for ARQ) or non-existent (blacklisted). From an energy consumption perspective, a link used by ARB consumes the same energy as a link under UDG. 


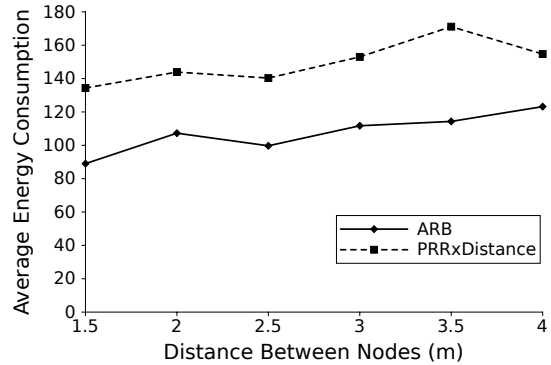

Fig. 3. The ARB strategy is more energy efficient than the PRR $\times$ distance metric, consuming between $25 \%$ and $50 \%$ less energy.

Although the second assumption is not accurate because the optimal links are of different lengths, we argue that UDG can nevertheless closely approximate ARB. This is because of the Gaussian nature of the relationship between PRR and distance. Over a large number of samples the average length of the optimal links should converge. If the radius of the UDG model is set to this average then UDG should closely approximate ARB.

As an initial test of this theory, Monte Carlo simulations of a chain topology were performed. The source and destination nodes were placed $1,000 \mathrm{~m}$ apart with nodes evenly spaced between them. The parameter values are as above with $q=$ 1.0. The distance between the nodes was varied to examine the effect of density. In the UDG model, the radius value was manually tuned.

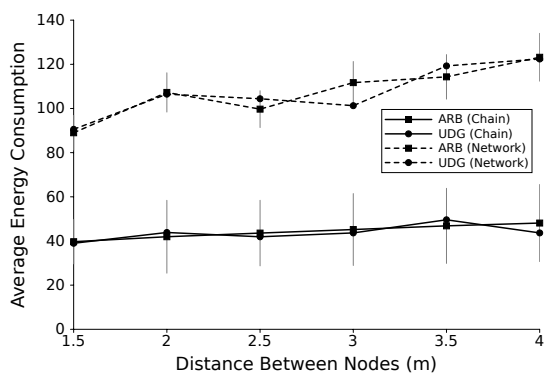

Fig. 4. The UDG model applied to a simple chain topology or a complete network is a close approximation to the optimal ARB strategy. Although at low densities the two diverge, UDG remains within the margin of error of ARB.

Fig. 4 shows the results for 50 chain topologies and shows that the UDG model is a close approximation of the performance of the ARB strategy. The discrepancy between the two is less than $5 \%$ for most densities, although the two models start to diverge as the density falls. Nevertheless, the UDG values are inside the margins of error of ARB which increase as density falls.

To confirm these results a full set of network simulations were conducted following the method described above. The results, also shown in Fig. 4, again show that UDG closely approximates ARB. Indeed, there is no statistically significant difference in their means $(p \geq 0.135)$.

\section{CONCLUSION}

Position-based routing is an important paradigm for wireless sensor networks. In this paper we have revisited the trade-off between hop-length and link quality. We have argued that a routing policy that excludes unacceptably poor quality links is more energy efficient because it can dispense with ARQ while other strategies, such as PRR $\times$ distance that allows the use of low quality links require ARQ. We have validated our claim through analysis and simulation showing that for a range of different densities, an absolute reception-based blacklisting (ARB) strategy is between $25 \%$ and $50 \%$ more efficient. However, this result depends on the relative sizes of data and control packets. It also assumes that the network is dense enough to ensure that at least one high quality link is very likely to exist.

This result allowed us to justify the widely used UDG model. We have argued that the UDG model is a close approximation of the performance of ARB because in both cases links are either perfect (no ARQ needed) or not considered for routing. Analytical and simulation results show that UDG can be consistently within 5\% of the performance of ARB.

In this paper we directly utilised the average length of the optimal links under ARB to select the radius of the disk under UDG. While this provided support to our argument that UDG can closely approximate ARB, it is a time consuming process that defeats the benefit of using the simpler model. In the future we would like to conduct a more thorough analysis of the relationship between the network parameters such as path loss exponent and density, and the disk radius that minimises the discrepancy between UDG and ARB. In this way we will be able to provide a method for translating a UDG model into its more realistic ARB equivalent.

\section{REFERENCES}

[1] K. Seada, M. Zuniga, A. Helmy, and B. Krishnamachari. Energyefficient forwarding strategies for geographic routing in lossy wireless sensor networks. In Proceedings of the 2 nd international conference on Embedded networked sensor systems, pages 108-121. ACM, 2004.

[2] J. Kuruvila, A. Nayak, and I. Stojmenovic. Hop count optimal positionbased packet routing algorithms for ad hoc wireless networks with a realistic physical layer. Selected Areas in Communications, IEEE Journal on, 23(6):1267-1275, 2005.

[3] M. Zuniga and B. Krishnamachari. Analyzing the transitional region in low power wireless links. In Sensor and Ad Hoc Communications and Networks, 2004. IEEE SECON 2004. 2004 First Annual IEEE Communications Society Conference on, pages 517-526. IEEE, 2004.

[4] S. Lee, B. Bhattacharjee, and S. Banerjee. Efficient geographic routing in multihop wireless networks. In Proceedings of the 6th ACM international symposium on Mobile ad hoc networking and computing, pages 230-241. ACM, 2005.

[5] I. Stojmenovic, A. Nayak, and J. Kuruvila. Design guidelines for routing protocols in ad hoc and sensor networks with a realistic physical layer. Communications Magazine, IEEE, 43(3):101-106, 2005. 